\title{
REFERENCE OF SENSATION AT THE SPINAL LEVEL
}

\author{
BY \\ P. W. NATHAN
}

From the Neurological Research Unit of the Medical Research Council, National Hospital, Queen Square, London

After the spino-thalamic tract has been cut in man, loss of sensation of pain and temperature caudal to the lesion is to be expected. In a small proportion of patients, although analgesia-in the usual sense of the term-is present, painful or thermal stimuli applied to parts of the body caudal to the lesion arouse a sensation, which is felt, not at the place actually stimulated, but in a normally innervated part of the body. Thus, a pin applied to the analgesic left leg, for instance, may cause a sensation referred to the normally innervated right leg.

This form of reference of sensation following the cutting of the spino-thalamic tract on one side of the cord was described by Ray and Wolff in 1945 . A somewhat similar phenomenon had previously been described by Obersteiner in 1881, and named " allochiria". In Obersteiner's cases, the phenomenon consisted of a difficulty in localizing the site stimulated rather than a definite reference. Obersteiner's reference of sensation consists of "the fact that though the sensibility is retained more or less completely, yet the patient is not clear, or is frequently, if not constantly, in error, as to which side of the body has been touched ". A condition more like that described by Ray and Wolff had been studied in monkeys by Mott in 1892 . He found that after a hemisection of the cord stimuli applied to one side were apparently felt as though they had been applied to the other.

In spite of these early observations this phenomenon has awakened little interest. Ray and Wolff, for instance, seem not to have known that a similar phenomenon had already been observed.

In my experience the reference of sensation described by Ray and Wolff is a part of a more general phenomenon. In patients with division of one spino-thalamic tract there may be reference not only to an analogous place on the opposite side of the body, but cranially, caudally, and in more complicated ways. Further, the reference may occur following division of both spino-thalamic tracts. It may also occur without the division of any tracts.
The purpose of this paper is to describe the features of such reference of sensation and to recom it in conditions other than in those previousfy reported. Certain related phenomena will also be described. An investigation of the various forrots of the reference of sensation will be reported ; and the possible underlying mechanism and the anatomical implications will be considered.

\section{Observations}

This reference of sensation has been observed in one patient having an amputation of an upher limb, in one patient with a thrombosis of $\frac{0}{\mathrm{~N}}$ tbe anterior spinal artery, and in 13 patients folloQiog antero-lateral cordotomy. The observations omthe amputation case, the case of anterior spinal a thrombosis, and six cases of cordotomy will given in some detail; the findings in the outher seven patients will not be given, as they are simitir to those of the other cases.

There are certain broad patterns of the mode off reference of the sensation. Any one form or a ky combination of the forms of reference may occdir in any one patient. There may be a reference froly the analgesic side to the analogous place on the opposite side of the body, there may be referente cranially or caudally to normally innervated pagss of the body, there may be a spread of sensation feit as coming from the place stimulated; there may also be a reference to one constant region of the body of all stimuli applied to the analgesic parts.

Case 39*.-An antero-lateral cordotomy was pe्दुformed on the left side of the cord at the second cervical segment. This produced analgesia throughout the right side of the body below the second and third dermatomes.

Forty hours after the operation it was observed that the sensation from a pin of $140 \mathrm{~g}$. weight was referred from one side of the body to the other. This was stpected by the examiner, for when the pin was applied to the analgesic right side, the patient immediately put fof his hand and covered an analogous place on the left side of the chest. When he was asked why he did this, twe

* The numbers of the cases are the same as those used throughert the series of papers by Nathan and Smith. 
replied that he did not know. On every occasion that he was pricked on the right side of the chest, he always put his hand up to rub the left side. Eventually he said that he could hardly believe his senses, but it seemed to him that he felt the pinprick applied to the right side of the chest as though it were on the left.

The following forms of stimulation caused this reference of sensation: pinprick of $140 \mathrm{~g}$. weight; pinching the skin; pressure with an algometer of $20 \mathrm{lb}$. weight ; a test-tube of water at $60^{\circ} \mathrm{C}$. (but not at $50^{\circ} \mathrm{C}$.) ; spraying the skin with ethyl chloride; squeezing muscles. The nature of the sensations induced by these various stimuli was not the same regardless of the nature of the stimulus and was not what would have been expected from the nature of the stimulus. Pinprick applied to the right side of the chest was felt on the left as the sensation of an electric shock on some occasions, on others as a pinprick; pressure with the algometer on the right felt like an electric shock on the left, only more painful; on other occasions it felt like painful pressure ; water at $60^{\circ} \mathrm{C}$. applied to the right side of the chest felt on the left either like pressure or "a soreness"; ethyl chloride applied to the right felt on the left like tickle or like cold ; pinching the skin on the right felt like a pinprick on the left; and pinching the whole thickness of the axillary fold felt on the left painful, " in the same way as tapping a bruise is painful ". The most striking feature of all sensations referred to the left was that they were felt to come from within. Stimuli applied to the right side of the chest were felt on the right as coming from without, in the normal way; but the stimuli applied to the right side of the chest were felt on the left as though the stimulus had been applied inside on the left; it came from within outwards. The patient could be made to have the sensation of pinprick on the left side by the pin being applied either on the left or the right; however he could always tell which side had in fact been stimulated, as when the left side was actually pricked he felt the pinprick coming from without, whereas when the right side was pricked, he felt the sensation on the left coming from within. When he was pricked with a 5-g. pin on the left, he felt this in a normal manner; when he was pricked with a 50-g. pin on the right, he felt this as touch on the right and as a pinprick coming from within on the left. These two stimuli were then applied simultaneously in analogous positions. The patient then felt on the left a pinprick coming from outside, and noticed nothing on the right. Thus the 5-g. pin on the left preponderated over the 50 -g. pin referred from the right. It was possible to deceive the patient by the use of bilateral simultaneous stimulation. For example, a touch stimulus applied to the left and a prick stimulus to the right gave sensations of pinprick on the left and of touch on the right. With suitably weighted pinpricks, bilateral simultaneous stimulation could be applied so that only touch was felt on the right and two pricks were felt on the left, the one coming from outside and the other from inside.

Fig. 1 shows the region of the body from which stimulation was referred to an analogous place on the opposite side. The upper limit was the second intercostal space. The nearer the stimulus was applied to this upper limit, the more easily it was referred; thus only a slight stimulus would evoke the referred sensation, and the referred sensation was felt more clearly and more quickly. Stimuli, however, were not invariably referred to the opposite side of the body ; on one or two occasions hard pressure applied by the algometer on the right side gave a sensation on the same side, referred cranially to the normally innervated segment of the neck.

Case 11.-An antero-lateral cordotomy was performed on the right side of the cord at the sixth thoracic segment and a similar one on the left at the seventh thoracic segment. For about three months following the operation there was analgesia to heavy pinprick throughout the left side of the body up to the lower border of the ninth thoracic dermatome and throughout the right side of the body up to the lower border of the second lumbar dermatome. After that time, the sensibility recovered on the right, so that there was no total loss of pain sensibility; the upper border of diminution of sensibility remained as before; when deep pressure was used as the painful stimulus, it was found that there was only hypoalgesia throughout the right lower limb. There was no change in the loss of sensibility on the left.

A fortnight after the cordotomy, pressure applied by the algometer was felt in the following way. When it was applied to the left buttock or the left tendo Achillis, the painful sensation was referred to an area in the left chest wall, about 6 in. caudal to the axilla; this was within the region of the girdle distribution of pain and tenderness which followed the operation. When it was applied anywhere on the right lower limb, it was referred to the region of the right patella; even when applied to the right popliteal fossa, it was still felt in the region of the patella, in front of the limb. Thus, on the right, the pain of the stimulus was felt in the knee, whether the stimulus was applied cranial (lumbar 1 and 2) or caudal (sacral, 1, 2, 3, or 4) to the segmental nerve supply of the knee. The patient said that these stimuli which were referred to the knee were referred to the identical place at which she had had pain years before. At that time she had been involved in an accident and had a painful comminuted fracture of the right patella. After the patella had been excised, the pain had gone, and she had forgotten about the whole incident. The reference of the painful pressure with the algometer, however, had produced the same pain in the same place, and had forcibly reminded her of the previous damage to her knee.

Placing her lower limbs in water at $60^{\circ} \mathrm{C}$. also caused reference of sensation. It caused a sensation as though the limbs had been put in electrically charged water. On the right this electric shock feeling shot to the knee; on the left it shot up to the girdle region of tenderness. There was no sensation of burning or warmth. This reference of the sensation is shown in Fig. 2.

Case 32.-An antero-lateral cordotomy was performed on the right side of the cord at the seventh thoracic segment. It produced analgesia throughout the left side of the body up to the lower border of the seventh thoracic dermatome. 
Fig. 1.-The single stippled area shows the region of analgesia. The doubled stippled area shows the region from which sensation was referred.
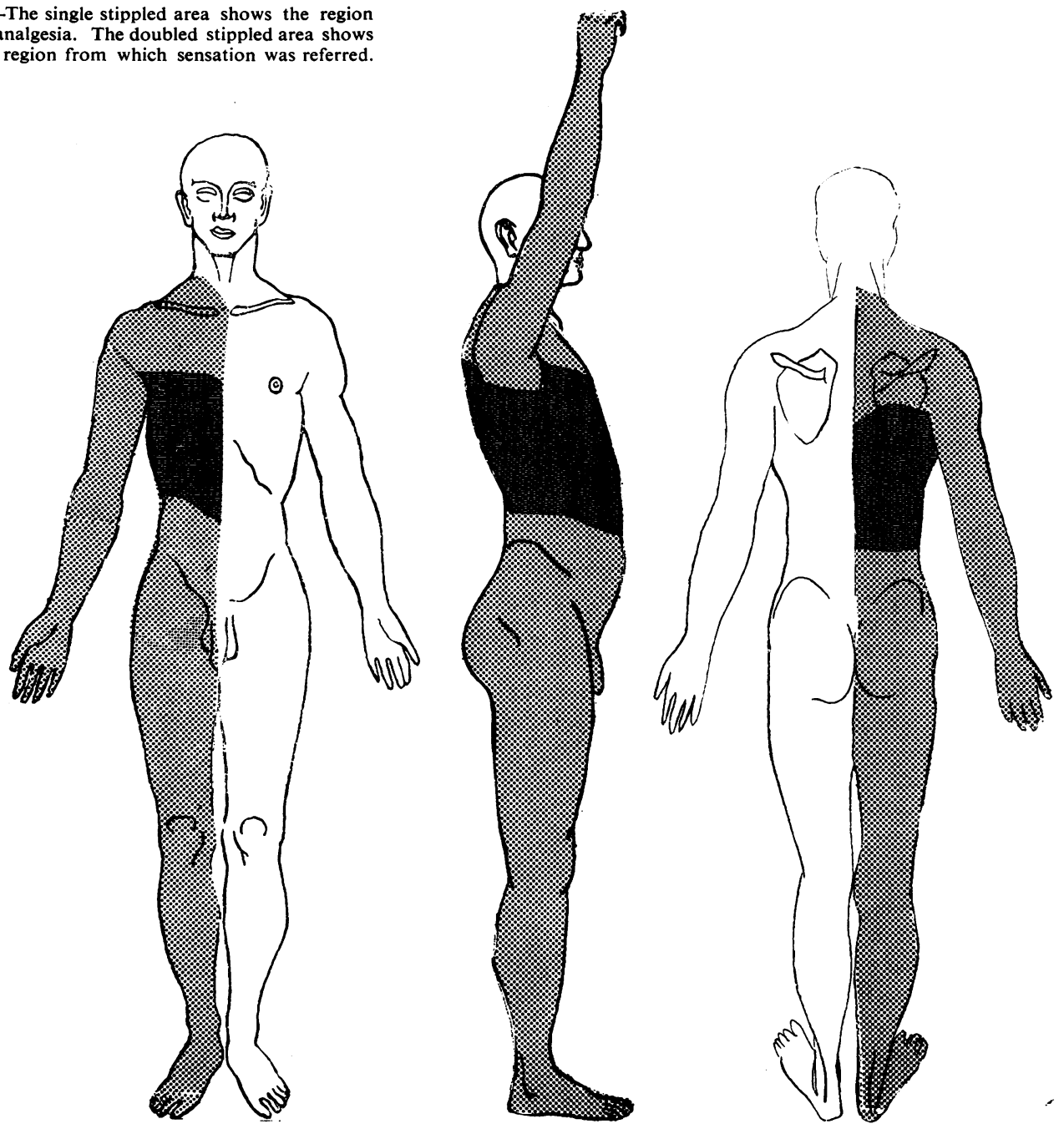

Twenty-four hours after the operation it was found that the sensation from a pin of $140 \mathrm{~g}$. weight was referred from the left groin towards the back or cranially ; locally this stimulus was felt as touch. Twenty-four hours later pressure with the algometer was referred from the back of the left thigh to the back of the right thigh, and from the left iliac crest cranially on the left to an area immediately cranial to the limit of the analgesia area. At the places where these stimuli were actually felt they felt like pinpricks; at the places where they were applied they were felt as painless pressure. On the next day it was observed that pins of 90 and $140 \mathrm{~g}$. weight applied just caudal to the inguinal ligament were felt as pinpricks just caudal to the left scapula.

By the tenth day after the operation the region of analgesia had diminished so that pinpricks of $140 \mathrm{~g}$. weight were felt above the tenth thoracic segment. If such a prick were applied at the place where this region of analgesia merged into the region of hypoalgesia abo胥, then it was felt as a prick both at the place at which it was applied and in the region of the left scapula. Cauda to this region pricks were felt locally as touch, while the pain was referred cranially to the left thoracic cage gin the axillary line.

A fortnight after the operation rapidly repeated hig pinpricks to the lower quadrant of the left side of the abdomen or to the anterior surface of the left thigh were felt in the same place in the left side of the chest immediately cranial to the upper boundary of the region $7 f$ hypoalgesia. It became apparent that there was a region on the left side from which all painful stimuli were most easily referred: this region overlapped the twelfth thoracic dermatome by an inch or so cranially and caudally; it stopped at the midline. Stimuli applfegd here caused "a strange feeling"- the patient could not say more than this. At the region to which the painful 
FIG. 2.-The stippled area shows the region of analgesia. The feet are shown in buckets of water at $60^{\circ} \mathrm{C}$.
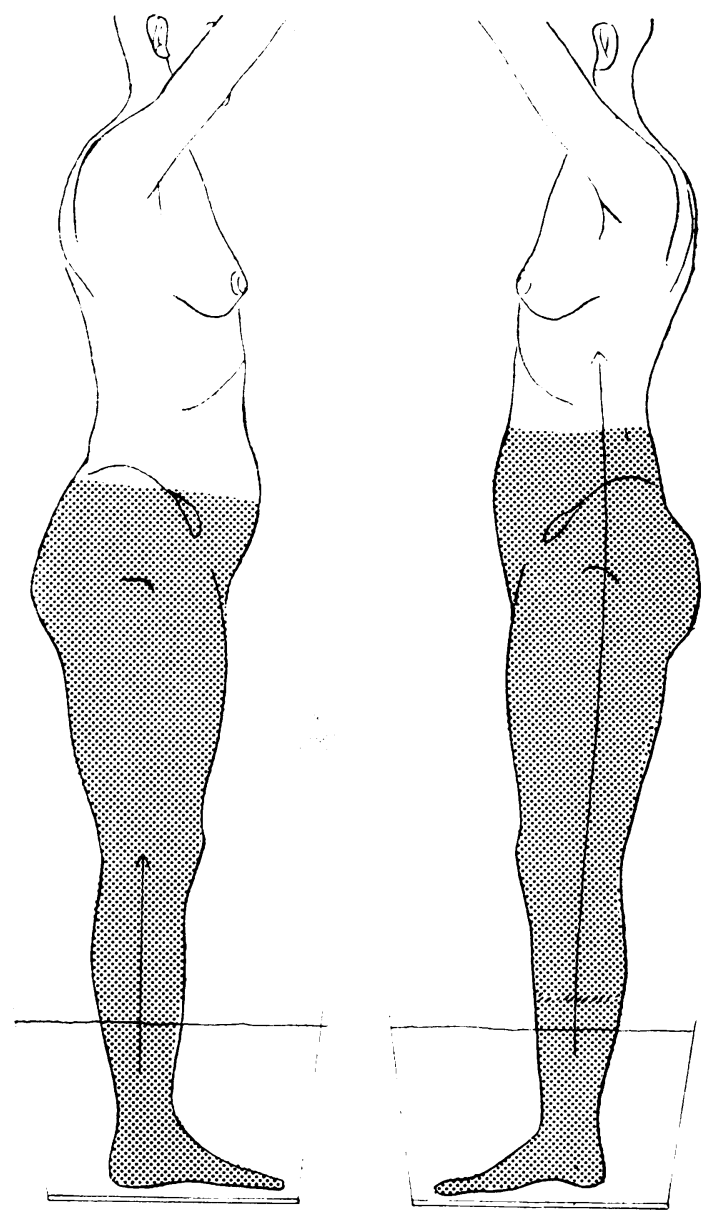

stimuli were referred, they felt like a pinprick, yet more diffuse and deep inside ; when the stimulus was repeated rapidly, it felt like a single continuous painful pricking. The reference of the sensation was not in the same plane cranio-caudally; on most occasions stimuli applied anteriorly were felt cranially in a more posterior plane.

Ten weeks after the first cordotomy, another one was performed on the other side of the cord at the second thoracic segment. Following this operation there was on the right side of the body very marked analgesia up to the lower border of the second thoracic dermatome; on the left side there was marked analgesia up to the lower border of the eleventh thoracic dermatome, and complete analgesia up to the lower border of the twelfth thoracic dermatome.

The manner in which repeated heavy pinpricks were referred is illustrated in Fig. 3. Stimuli applied to the territories supplied by the lower lumbar or the sacral nerves were not referred. The region to which the stimulation was referred was usually a constant area ; it was a band running cranio-caudally immediately posterior to the anterior axillary linc. Eventually there developed at this region a spontaneous tingling, present all the time; and this region became tender to pressure.

One month after the second operation, $400 \mathrm{ml}$. of fluid was run by drip into the patient's bladder. As the amount and the pressure increased, she got the pain in the usual place over the left ribs ; when the bladder was emptied, this pain was relieved.

About six weeks after the second operation all stimuli giving rise to impulses normally conducted by the spinothalamic tract produced a sensation in this same area in the left side of the thoracic cage. The reference was most easily obtained ipsilaterally, a stronger stimulus being needed on the right to cause it. Usually the stimulation was felt as running up to this region in the left side of the chest ; for example, if pressure was made with the algometer, it was not felt as long as the pressure was very light ; as the pressure was increased, the sensation was felt travelling up to the region to which sensations were referred ; further pressure produced more intense pain in this region; it did not travel further cranially. The reference was most easily obtained by using a cold stimulus, such as ethyl chloride spray; it was also obtained by pinprick, algometer, the application of hot water, or by distending the bladder; it was not obtained by injecting $5 \%$ saline.

Two weeks later cystometrography was repeated. After $300 \mathrm{ml}$. of fluid had been run into the bladder, $0.25 \mathrm{mg}$. of carbaminoylcholine was injected intramuscularly. As the pressure slowly rose, she got the pain in the usual place in the left side of the chest. The pain started as the pressure reached $25 \mathrm{~cm}$. of water; at a pressure of $31 \mathrm{~cm}$. it was severe. These pressures were associated with only 250 to $300 \mathrm{ml}$. of fluid in the bladder. The sensation felt in the left side was " a painful twisting feeling ". It did not go off immediately the bladder was emptied. Thus a quite usual amount of fluid in the bladder held at a physiological pressure caused pain referred to the constant region. Stretching the anal ring only slightly more than might normally occur brought on a pricking pain in the same place on the left side.

An experiment was carried out consisting of anaesthetizing that region to which sensations were referred. Following the injection of $8 \mathrm{ml}$. of $1 \%$ procaine, there was no longer any local tenderness. As long as this region remained anaesthetic, there was no reference of sensation. This experiment was controlled in two ways : on another occasion $8 \mathrm{ml}$. of $1 \%$ procaine was injected into the buttock; reference of sensation occurred as usual, showing that this amount of procaine had no general effect in stopping the reference of sensation; on another occasion, the tender area was injected with normal saline, the patient being told that it was the same experiment as before ; however, on this occasion, there was the usual reference of sensation.

The area in the left side of the chest to which the sensation tended to be referred was in itself not apparently abnormal, apart from being somewhat tender to pressure. No abnormalities were revealed by radiography, and at necropsy nothing abnormal was found. 
Case 38.-An antero-lateral cordotomy was performed on the right side of the cord at the third thoracic segment. It produced some impairment of pain sensibility up to the lower border of the third thoracic dermatome, much impairment of pain sensibility up to the fifth thoracic dermatome, and total analgesia to all forms of painful stimuli up to the lower border of the eleventh thoracic dermatome on the left.

About a fortnight after the operation the patient gradually became aware of an uncomfortable sensation over the posterior part of the right ilium. This developed so that it eventually became the identical pain that she had had on the left before the operation, felt in the same region but on the opposite side of the body. It was not due to any sudden spread of the cancer involving the nerves or the bones on the right. Five weeks after the operation it was found that pressure with the algometer on the anterior, lateral, and posterior aspects of the left thigh, and in the left popliteal fossa caused a reference of sensation to an area on the posterior aspect of the right ilium : this region was that where she had previously complained of the spontaneous pain developing after the operation. Over the next few days this spontaneous pain became more severe, and its area became more extensive, so that it involved also the right thigh. At this stage, all large active or passive movements of the left lower limb caused pain referred to the painful area on the right; the movements most likely to cause this referred pain were flexion of the lowerspine, and flexion, extension, and adduction of the left lower limb. It is to be noted that although such movements may well have stretched the right sacro-iliac joint or the attachments of the ilium to the sacrum, any movements of the right lower limb designed to cause pain in the ilium failed to do so ; thus we had the paradoxical position of being able to cause pain in the right iliac region by large movements of the left lower limb, whereas we could not cause pain in the right iliac region by similar movements of the right lower limb.

The experiment was performed of anaesthetizing with $1 \%$ procaine the area to which pain was referred. However, it was unsuccessful, in that the whole region, which by this time took in the whole of the inguinal region and the right ilium, was not rendered anaesthetic. Following the injection, the reference of the stimuli
FIG. 3.-The stippled area shows the region of analgesia.

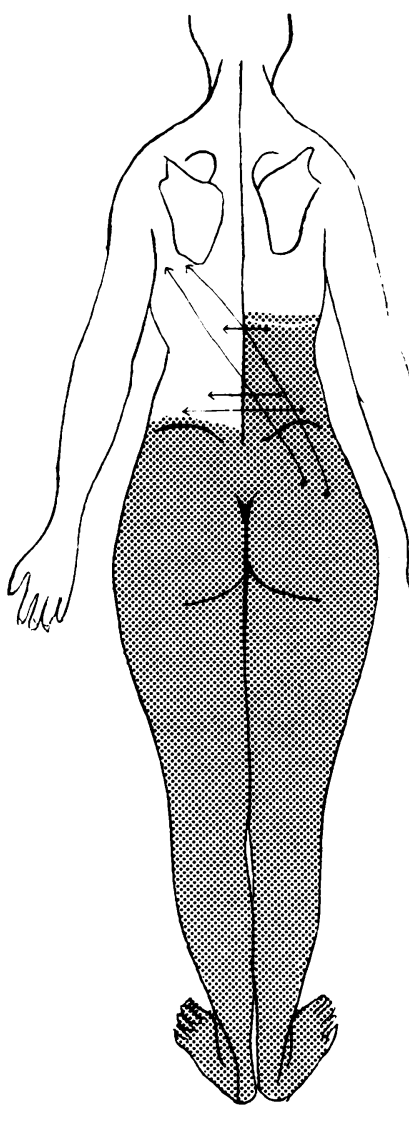

occurred largely to those regions that had not been properly anaesthetized. Pain from large movements $\vec{\delta} f$ the spine at the left lower limb was still referred to the deeper parts of this region that remained unanaesthetized.

Once this reference of sensation had become esta्ablished, it gradually became easier to elicit it. At firstit needed at least $40 \mathrm{lb}$. pressure with the algometer cause it ; eventually it appeared with only $20 \mathrm{lb}$. . A t first stimuli were referred only when they were appliged to the totally analgesic region; eventually stimuli werre referred when they were applied to hypoalgesic regions, such as the left side of the abdomen. Such stimuli were thus referred across the midline and caudally. stimuli applied to the right normally innervated side of the body were referred.

Twelve weeks after the first operation a further antero-lateral cordotomy was performed on the left sole of the cord at the sixth thoracic segment. It produçd some impairment of the sensibility of pain up to lower border of the fifth thoracic dermatome, axpd complete analgesia below the lower border of the 
lumbar dermatome. Painful pressure in the most cranial part of the totally analgesic area on the right was now referred upwards to the partially analgesic area on the right, just above the inguinum and around the iliac crest. This second operation removed all her spontaneous pain, and it was no longer possible to get reference of pain to the right from stimulating any part of the body on the left.

Case 54.-An antero-lateral cordotomy was performed on the right side of the cord at the first cervical segment. It produced complete analgesia throughout the left side of the body up to the lower border of the fourth cervical dermatome, and hypoalgesia up to the lower border of the fifth cranial nerve territory.

Within three days of the operation it was found that painful pressure applied to the analgesic area was felt as though it came from an identical spot on the opposite side of the body. The following forms of stimulation caused a reference of sensation to the normally innervated side : painful pressure ; pinprick ; slight pinching, just enough to cause pain in normal areas; smacking, also just enough to cause pain; stretching the skin by fixing it firmly and then dragging a finger firmly along the stretched skin. Spraying with ethyl chloride in this case did not cause reference of sensation. The region from which stimulation was referred extended from the second rib above to the knee below. On the right side of the body, at the places where the sensations were felt, the nature of the stimulus felt always the same, regardless of the actual kind of stimulation; the patient stated that had he got only the right side of the body from which to judge the nature of the stimulus, he could not tell what was the nature of the stimulus. On the right stimulation felt like " a tearing kind of pricking" and it felt as though it came from within outwards. The reference of the sensation was roughly to the same place on the right side of the body, but it was not always exactly the analogous place. Stimuli applied to some areas on the left were referred to quite a different place ; for instance, squeezing the left abdominal wall gave a pain in the region of the bladder on two occasions ; on other occasions it was referred to the analogous place to where it was applied on the right abdominal wall. Squeezing the left thigh on one occasion gave a sensation in the right thigh, but on another occasion it gave the feeling that he needed to micturate.

An experiment was performed of inducing a tender and painful area on the right side, for it was desirable to know if a painful area could mop up-so to speakstimuli from the other side. A quarter of a $\mathrm{ml}$. of $6 \%$ saline was injected into the right pectoralis major; this caused local pain. The various kinds of stimulation which previously caused reference were then applied to the left side of the chest. It was found that they were not referred to the tender and painful area on the right, unless they were applied in the same dermatome on the left. This experiment was repeated, the saline being injected on this occasion into the right rectus abdominis. Stimuli were not referred to this region of induced tenderness. Thus it was concluded that an area artificially
FIG. 4.-The stippled area shows the region of analgesia.

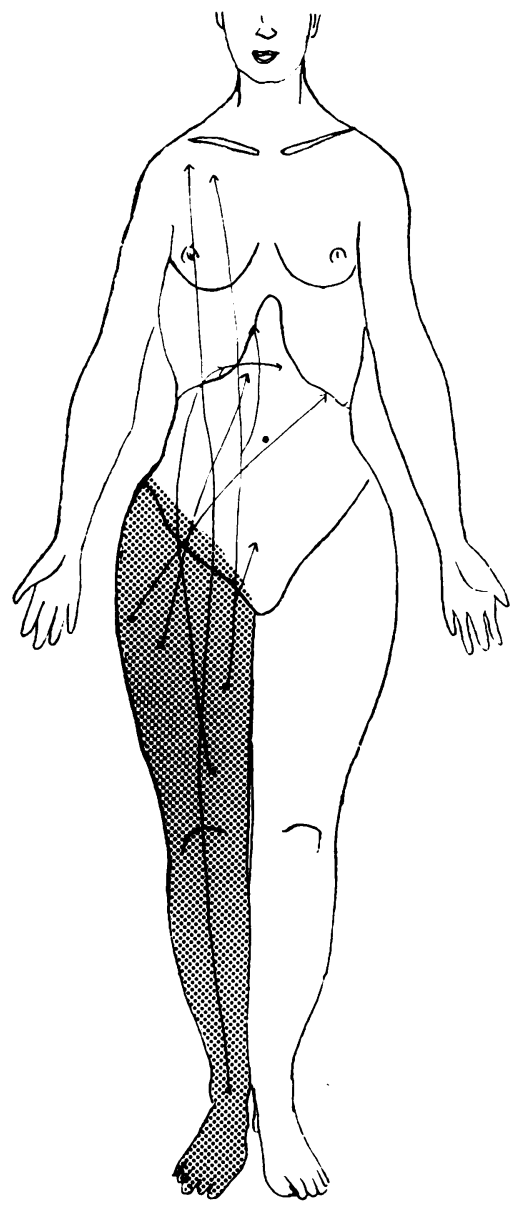

and temporally made tender did not serve as a region to which painful sensation was referred.

Case 41.-An antero-lateral cordotomy was performed on the left side of the cord at the sixth thoracic segment. It produced total analgesia throughout the right side of the body up to the lower border of the twelfth thoracic dermatome, and hypoalgesia up to the lower border of the tenth thoracic dermatome.

Before this operation the pain had been throughout the right lower limb and of a deep aching and burning kind ; after this operation, this identical pain was felt in the left lower limb. The patient observed this shift of the pain from one limb to the other as soon as she came round from the anaesthetic.

No reference of sensation was found when heavy pinpricking was used; but painful pressure with the algometer caused reference of pain. The stimulus was felt to spread in a diffuse unlocalized manner, illustrated in Fig. 4., as an unpleasant form of tingling. Many of the stimuli were felt in the right hypochondrium, and 
travelling from here to the epigastrium and the left hypochondrium; this was the region where she had the common girdle pain that occurs after the operation. Hyperextension and rotation of the right foot caused reference of the pain; it caused a tight feeling where the girdle pain was situated, and it also caused pain in the region of the trochanter of the left femur, that is, in a region identical with that in which she had previously had the pain on the right for which she had the operation.

Case 9.-A thoraco-lumbar sympathectomy was performed in this patient in two stages. During the second operation, performed one month after the first, the patient became very shocked. When she recovered from the anaesthetic, it was apparent that she had a thrombosis of the anterior spinal artery. This diagnosis was eventually confirmed histologically 10 months later.

There was analgesia below the lower border of the twelfth thoracic dermatome bilaterally. All pricks of $140 \mathrm{~g}$. weight or deep pressure applied with the algometer were referred cranially to the normally innervated parts of the body. In this patient no stimuli were referred across the midline. For instance, pressure applied to the anterior surface of the lower part of the right leg was felt in the inner part of the right inguinal region; and pressure at the same spot on the left was referred to a place just to the left of the umbilicus. Pressure applied to the right calf was felt in the right breast, and pressure to the back of the right thigh was felt just below the right breast. Pressure on the back of the left thigh was felt just below and to the left of the umbilicus. Pressure just below the middle of the right iliac crest was felt just above the iliac crest, on the right, posteriorly. The principle of the reference was that the more severe the stimulus, the further cranially it was referred; and when the stimulus was applied gradually and slowly, it was felt as travelling up the body gradually and slowly. Although the stimulus was not referred across the midline, it was not felt necessarily in the same vertical plane. At the place at which the sensation was felt it had the character of a deep pinprick, regardless of the nature of the actual stimulus.

Case 62.-This man, in May, 1953, had an amputation of the left upper limb. Within nine months of the operation he developed intense pain and involuntary movements in the stump. This pain consisted of a burning ache deep inside the stump ; in addition, there was on certain occasions, the sensation as though boiling water were dripping on to him ; when this pain continued for long, he would get nauseated and vomit.

I first saw the patient in March, 1955. He was more or less free from pain when he was sitting naked, there being nothing touching his stump or the upper part of his body on the left; but the slightest touch or even a puff of air on the front of the chest on the left gave him severe pain. He had himself found that touches anywhere on the left above the level of the umbilicus were immediately felt above the left nipple; simultaneously he would get a shooting forward of the stump, and the pain of the boiling water being dripped on to it.

I found on examination that many stimuli were referred to an area above the left breast, mainly in that region of skin forming the border between the fift cervical and the third thoracic dermatomes. ThE referred sensation was felt as something burning hot an moving inside; a single stimulus would be felt at the place of reference for 20 to 30 minutes; if successive stimuli were applied, he could not feel them individually at the place to which they were referred, having the a sensation of continuous burning movement. The stimuli referred were not necessarily of a noxious character. For instance, touches applied to the right: breast with a nylon No. 1 hair were clearly felt on the lef as little burns. Touches on the stump with a nylon No. hair were felt on the stump as little pinpricks, and io the left side of the chest as little burns. All stimuli thas. were referred gave the sensation of a little explosion of burning, coming from within outwards. The place from which stimuli were referred were the left side of the face, both sides of the neck, the stump on the lefes the right side of the chest, and both sides of the abdomeno There was a gradation of ease of reference ; on the face ${ }_{2 .}$ the stimuli had to be more severe on the forehead thafis on the cheek or mandible in order to be referred; on the abdomen, they were more easily referred from the left side than the right; of all places, they were most easily referred from the posterior part of the stumpeo There was no reference of sensation from the lower limbsiv Stimuli that are normally painful were not those nitsos easily referred: spraying the skin with ethyl chlogde (a stimulus purely of cold) most easily caused refereree? and at the place to which it was referred, it caused mere pain than any other form of stimulation.

In addition to this reference of sensation, there over the front of the left chest, at the place to whicks sensations were referred, a great lowering of threshoj $\$$ for all forms of stimulation, and also a perversion of sensation so that even the lightest touches evoked pain For example, on the right chest, touches with nylow hair No. 1 (weight, 0.004 g.) were felt 16 of 20 applica tions, the patient being often uncertain whether he fel⿱ them or not ; on the left side of the chest, he felt 20 oun of 20 applications, and he felt them easily ; but on the left they were felt as little burns, and not as touches. Moving the axillary hairs on the left caused local burning pain and touching the stump with a nylon No. 3 hair gave local sensation of pinprick (as well as the reference mentioned above). Such stimuli as these cannot have stimulated "pain nerve endings"; one must conclude that stimulation of "touch endings" may cause this burning pain.

The experiment was performed of inducing a smali local region of temporary severe pain. One-third ml. o迎 $6 \%$ saline was injected into the ligaments of the spine below the seventh cervical vertebra. It caused no local pain, but increased the pain in the stump and in the regiores in the front of the left side of the chest to whichsensations were referred; it did not change the references when stimulation was applied to other regions ; ethy chloride, for instance, sprayed on the right side of the chest, the neck, or the left side of the abdomen, was still felt in the left side of the chest. 
The sympathetic chain was injected on the left. It caused a Horner's syndrome and vasodilatation on the left side of the head and neck. This injection with procaine reduced the spontaneous burning to slight, and took away the spontaneous jactitation of the stump. It did not stop the reference of sensation, although it removed its sensitivity and the ease with which it could be obtained. This injection was controlled on another occasion by injecting saline instead of procaine; this had no effect whatsoever.

On a further occasion the left supraclavicular nerves were injected with procaine. This reduced the severity of the pain in the left side of the chest, but the stump remained as sensitive as before. There was no reference of sensation to the anaesthetic area ; but there was still reference to the area caudal to the anaesthetized region; also, the region deep to the anaesthetized skin remained tender. The blocking of the sympathetic chain was far more effective than the blocking of the supraclavicular nerves in alleviating the pain and in reducing the reference of sensation.

\section{Summary of Observations}

The material presented here consists mainly of cases of division of the spino-thalamic tract. There was also one case in which there was no gross anatomical lesion of the tracts of the central nervous system, but an amputation of a forelimb ; this may be seen as an example of a complete peripheral nerve lesion. The 13 cordotomy cases were the only ones of a series of 71 cordotomies that showed this reference of sensation. This selection of material does not indicate the occurrence of the phenomenon in neurological material ; it merely reflects the kind of material studied.

The features of this reference of sensation will now be summarized. The case of thrombosis of the anterior spinal artery will be considered together with the group of cordotomies, unless the case is mentioned specifically.

There are two differences between the reference of sensation as seen in the cordotomy cases and as seen in the case of forelimb amputation. In the cordotomy cases, only those stimuli that set up impulses conveyed by the spino-thalamic tract were referred; such were pinching or pricking the skin, painful pressure with an algometer, squeezing muscles, causing painful movements of joints, the injection of hypertonic saline, distension of the bladder, contraction of the bladder caused by the injection of carbaminoylcholine, stretching the anus, applying ice to the skin, spraying the skin with ethyl chloride, applying hot water to the skin. In the amputation case, all forms of stimulation gave rise to referred sensation. In the cordotomy cases stimuli were referred only when they were applied to the region of the body that had been rendered largely or totally analgesic; in the amputation case, stimuli were referred when applied to parts of the body that were fully innervated.

From the histories given above it will be seen that the sensation might be referred to one constant place, or it might be referred in other ways ; and in any one case more than one mode of reference might occur. In those patients in whom there was no constant region to which sensation was always referred, the sensation was either referred to an analogous place on the opposite normally innervated side of the body, or to regions above or below the region rendered analgesic by the cordotomy, on the same side of the body. In some cases the sensation might be felt at the place to which it was referred immediately ; in others there was a gradual spread of sensation from the place stimulated. In the cases where there was a constant region to which stimuli were referred, there might also be a reference elsewhere than to this place. In some cases no cause for the selection of a specific region to which sensations tended to be referred was found; the actual region showed no pathological change ; and at necropsy no abnormality was found in the local tissues or in the relevant segment of the cord. Sometimes, however, the sensation was referred to the region of girdle pain which often follows the operation of cordotomy. In other cases the sensation was referred to an analogous place on the opposite side of the body to that where the pain had been before the cordotomy. In one case (Case 11) the region to which sensations were referred had some years previously been the seat of a traumatic process, and the reference of stimuli caused the identical pain in the identical place that had been experienced before. Sometimes there were two areas to which sensation could be referred (as in Case 11) ; these were on the two sides of the body, and each area attracted ipsilateral stimuli. When there was a constant area to which sensation was referred, sensations were referred there whether the stimulation was caudal to, cranial to, or across the midline from this area.

The regions from which sensation was referred were not identical with the hypoalgesic or analgesic regions. Reference of sensation was more likely to occur when the region stimulated was close to the place to which it was referred; similarly, the heavier or more severe the stimulus, the further away from the place of reference could it be applied and still referred. In some cases limits could be determined beyond which reference of sensation could not be induced. No part of the body, however, was found that did not in some patient give rise to this reference. On most occasions the stimulus was 
felt as travelling from the place of application towards the place where it was felt; when the stimulus was applied slowly, this slow travelling of the sensation could be appreciated; when it was applied quickly, it might be felt immediately at the place to which it was referred.

When the region to which sensation was referred was rendered completely analgesic, either by the injection of local anaesthetic solution or by another cordotomy, sensation was no longer referred to this region. An artificial, temporary area of tenderness, induced by the injection of concentrated saline, did not form an area to which sensation was referred. At the place where sensation was felt, the various forms of stimulation mostly caused an identical sensation. But this was not always so : in Case 1 various forms of sensation were experienced, although they were not necessarily those that would be expected from the nature of the stimulus. A further characteristic of the referred sensation was that this sensation was felt as coming from within outwards. Further, the sensation did not have the proper temporal relations of the applied stimulus ; it was always felt as prolonged, and it might continue for minutes after the stimulus had been removed; this characteristic resulted in the patient being unable to tell how often or when a new stimulus was applied. The place to which sensation was referred was always within a normally innervated part of the body. Thus in the cordotomy cases it was usually cranial to the hypoalgesic zone ; in those cases in which there was a normally innervated region caudal to the analgesic zone, reference might be to this caudal region. When there was no region to which sensation was constantly referred, it was apparent that the distance between the point of stimulation and the place to which it was referred was related to the grade of the stimulus : a slight stimulation would be referred just cranial to the analgesic zone, and a strong stimulation might be referred well into the cervical segments.

This reference of sensation can occur with lesions that divide the spino-thalamic tract in the medulla oblongata ; for, although I have had no opportunity of studying the patients, I know of two cases of thrombosis of the posterior inferior spino-cerebellar artery in which it occurred. There is no evidence that it occurs with total division of the spinal cord. The kind of reference of sensation called allochiria has been recorded since Obersteiner's description of it, with lesions of the posterior columns and of the cerebrum ; it will not be discussed here.

\section{Discussion}

These observations will be discussed in the following way: first, the pathway taken by the
FIG. 5.-Diagram of caudal end of spinal cord. The stippled region indicates that part of the cord rendered necrotic by the thrombosid of the anterior spinal artery in Case 9.

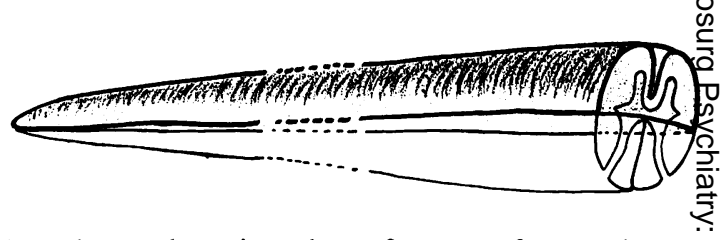

impulses subserving the reference of sensation te reach the neurological substratum of consciousness? will be considered; secondly, the mechanism of the various features of the reference will be considered 홓 thirdly, these two aspects of the subject will be correlated; and fourthly, other relevant apecto of the subject will be discussed.

What pathways do the impulses take to reach the neurological centres of consciousness? Here the. case of thrombosis of the anterior spinal artery $\overrightarrow{\mathbf{6 0}}$ particularly important. The extent of the cor\& rendered necrotic by this vascular occlusion is shown in Fig. 5. It is clear that, at least in this case, the relevant pathways must lie in the posterior halp of the cord.

The posterior columns need to be considered $\frac{\mathbb{1}}{\alpha \rightarrow q}$ possible pathway. These columns contain two ty of ascending systems : extrinsic fibres, with cello of origin in the posterior root ganglia; and intrins fibres with cells of origin in the grey matter of $\frac{\text { ofhe }}{3}$ posterior part of the cord. It is unlikely that pathway consists of fibres of the extrinsic system It is unlikely, for the reason that only that part of the sensation subserved by impulses normal conducted by the spino-thalamic tract is referred the part subserved by impulses normally conducted by posterior column fibres is felt in a normal manner. For instance, when painful pressure with the algometer is referred, the touch and the pressure are felt normally and correctly localized; it is the pain component that is referred. Two differeng modalities of sensation cannot be subserved by one and the same fibre (as far as is known). Further, is unlikely, for the reason that such a hypothesis would account for none of the features of this phenomenon, other than the patient's inability feel pain when the spino-thalamic tract was divide\& It would be expected that the pain would be felt $\mathrm{m}$ the same place as the touch or the pressure stimulus? there would be no reference of the sensation.

It remains to consider chains of short neurons: This hypothesis is certainly not a new one. In 1899 Münzer and Wiener performed the following experiment in the rabbit and the dog. They carrieg out complete transverse sections of the spinal cofd and also divided the posterior roots bilateral 
between the ganglia and the cord. After waiting for the posterior root fibres to degenerate, they stimulated the relevant area of the posterior columns; and they obtained evidence of typical pain reactions from the animal. The only intact fibres of the posterior columns could be intrinsic fibres. Rothmann (1906) performed a different experiment in the dog. He found that after the lateral, posterior, and anterior columns had been divided at different levels, the dog still manifested evidence of sensibility in the parts of the body from which the known afferent pathways had been removed. He concluded that sensory impulses reach the brain "via many short intraspinal tracts" which together form " a conduction chain". Karplus and Kreidl (1914, 1925) proved by carrying out hemisections on opposite sides of the cord of the cat that impulses subserving pain sensibility are conducted not only via long tracts but also through the grey matter. As the cats gave evidence of feeling pain immediately after the operation, Karplus and Kreidl were inclined to regard conduction "via a chain of short tracts" running within the grey matter as a part of normal conduction. This work was later confirmed by Kletzkin and Spiegel (1952).

As it seems that one may have to postulate a similar conduction of impulses subserving pain sensibility in man, one may ask where such short tracts might lie. Apart from being able to state that some of them are situated in the posterior half of the cord, we have no evidence on which to base an answer to this question. They might be any of the intrinsic fibres of the cord surrounding the grey matter, they might be similar fibres in the posterior columns, they might be fibres of Lissauer's tract, and they might be fibres within the grey matter itself, either within the posterior horn or deeper in the substance of the grey matter.

It is reasonable to suppose that one of the characteristics of conduction via chains of short neurons might be the loss of the correct local sign. An inevitable feature of long tract conduction is that the signal is not interfered with on the way ; it can be influenced only at the synapses. Indeed, it may be that this insulation from the surroundings is that which enables long tracts to convey local sign. But if the message runs from local station to local station, then at each station it might acquire the local stamp and its original stamp be lost. If the impulses travel along short chains of neurons each group of these might add its own local sign; then the sensation would be felt as passing along the body segment by segment, and felt finally at the segment corresponding to the last group of cells stimulated.
There are other features of conduction along such a chain that might be expected. A long fibre gives out at one end the same number of impulses as it receives at the other. But if there is conduction along chains of neurons, with many synapses on the way, then at each synapse the number of impulses might well be scattered and diminished. Each next link in the chain would then get a diminished input signal ; and the end station might well receive an output signal less than the original input. In such a system there might be a tendency for the message to become fainter or even to die out. This form of conduction would then fit in with one of the features of the reference of sensation : a slight stimulation is conducted a short way; stronger stimulation is conducted further.

One more supposition will be made: let us suppose that the short neurons concerned are neurons connecting the cells of origin of the spinothalamic tract. In such a case it would be expected that the sensation would have a painful character, or the character of heat or cold. But as this short chain type is not the normal mode of conduction, it might also be expected that there would be features other than those found in the normal. It would not be surprising to find that the sensation is felt as though it comes from within, and to find that its quality differs from that to be expected from the nature of the stimulus, and that its quality tends to be the same regardless of the nature of the stimulus. There being chains of neurons connecting the cells of origin of the spinothalamic tract caudally and to the opposite side of the cord as well as cranially, it would be expected that sensation would be referred caudally and to the opposite side of the body. When the impulses run caudally, they could be conducted to the neurological substratum of consciousness only where there were intact spino-thalamic fibres; in other words, the sensation could be referred caudally only where there was a region of normal or almost normal pain sensibility below the analgesic zone.

Thus the hypothesis of the reference of sensation depending on conduction via chains of neurons fits the facts fairly well. It accounts for all the features of the spread and the pattern of the reference; it accounts for the spread across to the analogous place on the opposite side of the body, the spread cranially or caudally to regions supplied by intact spino-thalamic fibres ; it accounts for the lack of local sign of that part of the sensation normally subserved by the spino-thalamic tract; it accounts for the fact that the reference is always to a region adequately innervated by the spino-thalamic tract, whether this is cranial, caudal, or on the opposite 
side of the body to the place stimulated; and it also fits in with the fact that the harder the stimulation, the further the sensation is referred, and the less severe the stimulation, the less far it is referred.

It is necessary to consider the mechanism underlying the use of such chains of neurons for the conduction of impulses subserving sensation. The main difficulty is to account for the occurrence of the reference of sensation in some cases of division of the spino-thalamic tracts and not in others. The case of amputation of the forelimb provides a clue. In this case there was no division of an afferent tract. But there was an abnormal state of hyperexcitability in the part of the central nervous system associated with the left upper limb; this was shown by an actual lowering of threshold, so that stimuli too light to be felt in normal regions were felt here ; and also by the stimuli being felt here for an abnormally prolonged time. It is suggested that the reference of sensation is associated with a state of hyperexcitability of the central nervous system, and that this state may be induced by division of a large number of nerves near the central nervous system or division of an algesic tract inside the central nervous system.

A state of hyperexcitability would cause a reduction of resistance at synapses; and this lowering of the normal resistance might well allow impulses to overflow into subsidiary pathways. It is possible that under normal conditions the great majority of impulses run along the usual tracts and that a small minority run in these subsidiary pathways ; or it is equally possible that under normal conditions no impulses travel via such subsidiary pathways, all the impulses subserving the various sensory modalities travelling in the posterior columns and spinothalamic tracts. It is suggested that in the cordotomy cases, where the reference of sensation occurs, the surgical lesion in the spinal cord sets up a state of hyperexcitability. In a sense, a lesion in the cord might be viewed as a switching mechanism, changing the course of impulses, so that they are directed entirely into subsidiary pathways. Why such a state of hyperexcitability should occur in some cases and not in others remains unexplained.

Let us now consider those cases in which there is a constant region (or regions) to which sensation tends to be referred. It can only be conjectured why there is such a region in some cases. For the parts of the body to which sensation was referred were examined after death and no pathological changes were seen and nothing abnormal was found in most cases in the relevant segment or posterior roots. It would seem to be a reasonable hypothesis to believe that in certain regions of the cord groups of cells have got into a persisting state $Z$ of hyperexcitability with a lowered threshold, that $\stackrel{\mathbb{Q}}{=}$ some change has occurred facilitating local synapticō transmission. In some cases one cannot say why such a persisting state would have developed; but in others, causes can be suggested. When a cancer(s) has been invading some peripheral nerves for⿳亠口冋 months, causing continuous discharge of impulses into one or two segments of the cord, one might imagine that all groups of these segments would become hyperexcitable. If the division of the spinothalamic tract is incomplete, the structures with aO nerve supply from these segments might remain somewhat painful and tender. Case 8 forms an $\frac{\bar{s}}{\overrightarrow{8}}$ example of reference to the previously very painful $\mathbb{Q}^{\mathbb{Q}}$ and later slightly painful area. If the division of the tract is complete, then the sensation is referred to the same segment, but on the opposite side of the body. The operation of cordotomy causes an area $\vec{\omega}$ of pain and tenderness cranial to the level of? hypoalgesia ; this region would seem to be associated with a local state of hyperexcitability. The total lesion of the brachial plexus in the amputationce case seemed to have set up a local area of hyper $-\int_{\infty}$ excitability on the left side of the chest at that plaseco where the cervical distribution meets the thora बco distribution. And finally there is the intriguigigcase where there had formerly been an injury to thes knee. It will be remembered that this patient hof years before broken the right patella, that she $1+\frac{1}{4} \mathrm{~d}_{0}$ had much pain at that time and that the pain kifd been taken away by removal of the patella ; the knee had become painless and the patient hado forgotten the whole matter. Yet many years later its was brought vividly to her mind by her experiencingo the identical pain in the identical place when hot $\frac{0}{\mathrm{D}}$ cold, or painful stimuli were applied to that side of the body. One has to conclude that such a damaged $\overrightarrow{0}$ and previously painful structure sets up a kind of $\overline{ }$ locus minoris resistentiae in the central nervous system; further that such a state can persist. unnoticed and causing no obvious effects for years and that its presence can be shown up by the state of hyperexcitability caused by an operation on the tracts of the spinal cord. Impulses passing this region of the cord under normal conditions do noto stimulate it ; but impulses passing it during a state of hyperexcitability or impulses taking abnormaf pathways do stimulate it.

Such a region of local hyperexcitability would be envisaged as firing off impulses into neighbouring neurons, some of which would reach the neurologica substratum of consciousness whenever a certain threshold is exceeded. It would be exceeded when $\omega$ ever the stimulating effect of impulses arriving 
within a certain effective period of time mounted up, or it might be exceeded spontaneously, there being enough local excitation to fire off the group of neurons. In the former case the region of the body supplied by these neurons would be painful all the time. In the latter case the region supplied might be tender or painful when a further aggregate of impulses were added from stimulating the local peripheral nerves and end-organs, or painful when a further aggregate of impulses were added from stimulating distant but connected regions. The impulses set up in these distant regions would have to pour into this hyperexcitable region, and not by-pass it, as occurs with normal long tract conduction. If the total number of impulses within a certain period of time at such a region of hyperexcitability were to be reduced, for example, by removing those arriving from local peripheral nerves (by means of a local anaesthetic block), then there might well be an insufficient accumulation of local stimulation to fire off the group of neurons ; in this case an additional quantity of impulses being poured into the region, originating from stimulation of the body elsewhere, might not be enough to bring the total quantity up to its firing-off threshold ; and then the sensation would not be referred to the region supplied by the hyperexcitable neurons. On the other hand, the region might be spontaneously so hyperexcitable that, in spite of cutting off the impulses arriving from local peripheral nerves, it would still fire off when impulses reached it from distant regions. When a fresh group of impulses arrive at such a region of hyperexcitability, it seems likely that they would increase the local excitability of the region for some time. - In this way, one stimulus referred to the place of hyperexcitability would cause sensation going on for some time; and a subsequent stimulus within that time might cause no new sensation. Such facts were observed.

If there are such groups of neurons in a hyperexcitable state liable to be fired off by passing impulses, why are they not fired off in the normal by impulses travelling along normal long tracts ? The answer, partly, might be that the normal tracts by-pass such groups of neurons. But when conduction is via short chains connecting the cells or origin of the spino-thalamic tract, when the pathway does not by-pass this region but runs right into it, the additional number of impulses would swell the local number sufficiently to fire off the region, giving rise to a signal along the spino-thalamic tract, having the local sign of that region. The other part of the answer to this question might be that such an area is fired off by passing impulses in normal tracts in some cases; such a case would be that of the painful amputation.
Where there is no such region of local hyperexcitability, then it seems likely that the impulses pass along the cord, from synapse to synapse, without firing off any one area in a massive way. In such a case reference of sensation is not to one constant region, but is to an analogous place on the opposite side of the body, or cranially, or caudally, or generally spreading diffusely from the point of stimulation.

Support for these hypotheses comes from experiments on animals. As has been mentioned above, in 1892 Mott observed the condition in monkeys, and in 1913 Dusser de Barenne made an experimental study of it in dogs. There is an outstanding difference, however, between the reference of sensation in the cases of cordotomy and that produced by hemisections of the cord in dogs, cats, and monkeys : in man the reference was from the side of the body contralateral to the incision (the analgesic side) to the ipsilateral side (the normally innervated side); whereas in the animals it was from the side of the body ipsilateral to the hemisection to the contralateral side of the body. However, this may not be such an important difference as it seems ; for there is a main difference in the functional anatomy of the algesic pathway in man and in these species : in man this pathway is totally crossed ; in these species it is not so. In the monkeys with a hemisected cord Mott found that " tested by heat, pricking and stimulation by faradism, it was very difficult to decide . . . whether the animal felt better on the side of the section or on the opposite side". He concluded that there is more sensory loss on the hemisected side than on the opposite side. With regard to the modalities of pain and thermal sense, it is clear that in the monkey and the dog a hemisection does not cause much loss on either side; it seems that in these species the spino-thalamic tract is more uncrossed than crossed. In comparing the reference of sensation observed by Mott in the monkey and that observed here in man, it seems then justified to neglect the relation of the reference to the side of the cord incised and to turn our attention entirely to the side of the body rendered analgesic or severely hypoalgesic. We then see that in man the sensation was referred from the analgesic side to the opposite side of the body (or elsewhere), and that in Mott's monkeys it was also referred from the most hypoalgesic side to the opposite side of the body. It is to be noted too that Mott found this reference of sensation in three out of nine monkeys operated upon, and that I found it in a minority of patients after cordotomy.

Dusser de Barenne (1913a) came across this phenomenon when he was studying the effect of strychnine on the spinal cord. He knew that the 
application of this substance to the dorsal region of one segment of one half of the spinal cord of the dog causes all forms of stimulation of the relevant segment to be felt as apparently painful. He then found that if he performed a hemisection of the cord a few segments cranial to the segment where he put the strychnine, he got paraesthesiae and hyperalgesia on the side opposite to the incision and the site of application of the strychnine. He made no mention of whether he ever found the phenomenon occurring without strychnine being applied. It is clear, however, that he obtained it in all cases in which strychnine was applied. He later (1913b) reported a further experiment on the phenomenon. On this occasion, in operating on a cat, he did not carry out a complete hemisection, but spared the posterior columns. He stated that the phenomenon could be obtained when the lesion was slightly more or slightly less than a hemisection, and he considered that all that was necessary with regard to the extent of the lesion was that it should divide unilaterally a cerebropetal sensory tract, and probably the algesic tract in particular.

Thus the hypothesis of a local region of hyperexcitability gains support from Dusser de Barenne's experimental production of a similar condition ; for he invariably produced the condition when he rendered the posterior root entry zone hyperexcitable.

Finally, it remains to observe that there is a practical everyday aspect of this phenomenon. The operation of antero-lateral cordotomy is a disappointing one. It is probable that the phenomenon described above is one of the causes of failure of such pain-relieving operations on the cord. The artificial stimulation applied to the body by deep pressure with the algometer or spraying with ethyl chloride serves to make manifest the stimulation that is acting all the time : stimulation from the invasion of nerve fibrils by cancer, the various stimuli acting spontaneously and intermittently, such as a full bladder, pressure of the weight of the body on a certain analgesic part of the body, a painless bedsore. The sensation from such stimulation may well be referred; in unilateral cordotomies it might be referred to an analogous place on the opposite side of the body or to the same part of the body as that where the pain was originally situated, but on the opposite side of the body ; in bilateral cordotomies, it might be referred vaguely upwards, or, where there is " sacral sparing" downwards to the region of normal or diminished pain sensibility, or it might be referred to a certain region outside the analgesic zone, a region which in itself is not] pathological and which may or may not be tender or it might be referred to the zone of girdle paim and tenderness which so often surmounts the zone of hypoalgesia, making this girdle pain continue foro weeks or months. It may well be that it is this mechanism of local hyperexcitability and reference. of sensation that accounts for the fact so oftef observed immediately after a unilateral cordotomy the presence of the pain on the side of the bod $\overrightarrow{x_{s}}$ opposite to the analgesic side, felt in an analogous place. For I have always paid particular attentio f5 to the laterality of the pain before the operation and have pressed the patients to admit that the have had some pain on the unaffected side; onlo when they have vigorously denied any trace of suckp pain have we carried out unilateral cordotomies $\overrightarrow{0}$ And yet we have seen an immediate transference of the pain to this normal side after the unilateraf operation. This has occurred just as frequently among those who before the operation denied an trace of pain on the normal side as among those who admitted that occasionally they had felt slight ache running across to the normal side.

\section{Summary}

A form of reference of sensation is describedz Although most of the examples of the syndrome occur after division, either surgical or natural, 의 the spino-thalamic tract or tracts, it may also ocouging
without any lesion of the long tracts.

The mechanism and relevant anatomical cone siderations are discussed.

I wish to thank Dr. E. A. Carmichael for his helpfug criticism, Mr. Wylie McKissock, who operated on Cases 39, 11, 32, and who allowed me to investigate Case 62, Mr. Murray Falconer, who operated on Cases 54 and 40, Mr. Bernard Harries, who operated on Case 41, and Dr. A. Barham Carter who allowed me tof investigate Case 9.

Cases 39, 11,32, 38, and 62 were in the Nationa Hospital ; their hospital case numbers are respectivel 14334, 23011, 29786, 36799, and 58071.

\section{REFERENCES}

Dusser de Barenne, J. G. (1913a). Folia neuro-biol., Lpz., 7, 549. (1913b). Mschr. Psychiat. Neurol., 34, 523.

Karplus, J. P., and Kreidl, A. (1914). Pflügers Arch. ges. Physiol $158,275$.

Kletzkin, M., and Spiegel, E. A. (1952). Fed. Proc., 11, 83.

Mott, F. W. (1892). Philos. Trans., B., 183, 1.

Münzer, E., and Wiener, H. (1899). Neurol. Zbl., 18, 962.

Münzer, E., and Wiener, H. (1899), N

Obersteiner, H. (1881), Brain, 4, 153.

Ray, B. S., and Wolff, H. G. (1945). Arch. Neurol. Psychiaß̧ (Chicago), 53, 257.

Rothmann, M. (1906). Berl. klin. Wschr. ,43,47, 76. 